\title{
Explicit Solutions of the Coupled mKdV Equation by the Dressing Method via Local Riemann-Hilbert Problem
}

\author{
Ting Su*, Guohua Ding, Zhiwei Wang \\ Department of Mathematical and Physical Science, Henan Institute of Engineering, Zhengzhou, China \\ Email: *suting1976@163.com
}

How to cite this paper: Su, T., Ding, G.H. and Wang, Z.W. (2016) Explicit Solutions of the Coupled mKdV Equation by the Dressing Method via Local Riemann-Hilbert Problem. Applied Mathematics, 7, 1789-1797. http://dx.doi.org/10.4236/am.2016.715150

Received: August 2, 2016

Accepted: September 18, 2016

Published: September 21, 2016

Copyright $\odot 2016$ by authors and Scientific Research Publishing Inc. This work is licensed under the Creative Commons Attribution International License (CC BY 4.0).

http://creativecommons.org/licenses/by/4.0/ The coupled mKdV equation

\section{Keywords}

\section{Introduction}

\begin{abstract}
We study the coupled mKdV equation by the dressing method via local RiemannHilbert problem. With the help of the Lax pairs, we obtain the matrix RiemannHilbert problem with zeros. The explicit solutions for the coupled $\mathrm{mKdV}$ equation are derived with the aid of the regularization of the Riemann-Hilbert problem.
\end{abstract}

Coupled mKdV Equations, Riemann-Hilbert Problem, the Dressing Method

$$
\begin{aligned}
& u_{t}-u_{x x x}+6 u v u_{x}=0 \\
& v_{t}-v_{x x x}+6 u v v_{x}=0
\end{aligned}
$$

is an important member of the AKNS hierarchy [1]. Moreover, it has various applications in mathematical and physical fields. In [2], Prof. Geng has given its quasi-periodic solution by using algebra-geometric methods. The equation can be solved by the method of the inverse scattering transformation, Hirota direct method, Lax pairs nonlinearization approach and others [3]-[6]. There are a lot of references for the topic [7][14].

In this paper, we study the Equation (1) with the help of the Riemann-Hilbert method following [15] [16]. The present paper is organized as follows. In section 2, we give the Jost solution of the spectral equation. In section 3, we discuss the analytic property of the Jost solution. In section 4, we give the Matrix Riemann-Hilbert Problem. In section 5, we obtain the soliton-solution of the coupled KdV Equation (2), and we drop 
the curve of the solutions with the aid of the Matlab.

\section{Jost Solution}

First, we consider the coupled KdV equation

$$
\begin{aligned}
& u_{t}=u_{x x x}-6 u v u_{x}, \\
& v_{t}=v_{x x x}-6 u v v_{x} .
\end{aligned}
$$

As is well known [2], the Equation (2) can be derived as the compatibility of the system

$$
\Psi_{x}=U \Psi, \Psi_{t}=V \Psi,
$$

where the $2 \times 2$ matrices $U$ and $V$ of the form

$$
\begin{gathered}
U=-\frac{1}{2} i k \sigma_{3}+Q, \quad Q=\left(\begin{array}{ll}
0 & u \\
v & 0
\end{array}\right) \\
V=\frac{1}{2} i k^{3} \sigma_{3}-k^{2} Q-i k Q^{2} \sigma_{3}+Q_{x x}+Q Q_{x}-Q_{x} Q-2 Q^{3}
\end{gathered}
$$

where $k$ is an arbitrary constant spectral parameter.

When $u=0, v=0$, we obtain the special solution of Equation (3). For convenience, we denote the special solution as $F=\exp \left(-\frac{1}{2} i k x \sigma_{3}\right)$. Then, the spectral Equation (3) is transformed into

$$
H_{x}=-\frac{1}{2} i k\left[\sigma_{3}, H\right]+Q H,
$$

where, $H=\Psi F^{-1}$.

In what follows, we study the Jost solutions $H_{ \pm}(x, k)$ of the Equation (6) satisfying the asymptotic conditions $H_{ \pm} \rightarrow I$, at $x \rightarrow \pm \infty$. Since $\operatorname{tr} U=0$, these boundary conditions guarantee that $\operatorname{det} H_{ \pm}=1$ for all $x$.

In fact, the Jost functions $H_{ \pm}$are not mutually independent. They are interconnected by the scattering matrix $S(k)$ :

$$
H_{-}=H_{+} F S F^{-1}, S(k)=\left(\begin{array}{cc}
a(k) & -\bar{b}(k) \\
b(k) & \bar{a}(k)
\end{array}\right), \operatorname{det} S(k)=1 .
$$

\section{Analysis Solutions}

Let us rewrite the spectral Equation (6) with the boundary conditions in the integral form:

$$
\begin{aligned}
& \left(H_{-}\right)_{11}(x, k)=I+\int_{-\infty}^{x} u(\xi)\left(H_{-}\right)_{21}(\xi, k) \mathrm{d} \xi, \\
& \left(H_{-}\right)_{21}(x, k)=\int_{-\infty}^{x} v(\xi)\left(H_{-}\right)_{11}(\xi, k) \exp [i k(x-\xi)] \mathrm{d} \xi,
\end{aligned}
$$

for the first column entries of the Jost matrix $H_{-}$.

It is easy to know that the exponent in (8) decreases for $\operatorname{Imk}>0$. The first column $H_{-}^{[1]}$ of the matrix $H_{-}$is analytic in the upper half plane and continuous on the real axis $\operatorname{Imk}=0$. Similarly, we know that the second column $H_{+}^{[2]}$ of the matrix $H_{+}$is 
analytic as well in the same domain. Then, we give a solution of Equation (6):

$$
\Omega_{+}(x, k)=\left(H_{-}^{[1]}, H_{+}^{[2]}\right) .
$$

It can see that it is analytic as a whole in the upper half plane.

The analytic solution $\Omega_{+}(x, k)$ can be expressed in terms of the Jost function. In view of (7), we derive

$$
\Omega_{+}=\left(H_{-}^{[1]}, H_{+}^{[2]}\right)=\left(a H_{+}^{[1]}+b(k) \mathrm{e}^{i k x} H_{+}^{[2]}, H_{+}^{[2]}\right)=\left(H_{+}^{[1]}, H_{+}^{[2]}\right)\left(\begin{array}{cc}
a(k) & 0 \\
b(k) \mathrm{e}^{i k x} & 1
\end{array}\right)
$$

with

$$
S_{+}=\left(\begin{array}{ll}
a(k) & 0 \\
b(k) & 1
\end{array}\right)
$$

In the same way,

$$
\Omega_{+}=H_{-} F S_{-} F^{-1}, \quad S_{-}=\left(\begin{array}{cc}
1 & \bar{b}(k) \\
0 & a(k)
\end{array}\right), \quad S_{+}=S S_{-} .
$$

It follows from the above formal as that

$$
\operatorname{det} \Omega_{+}(x, k)=a(k) \text {. }
$$

In what follows, we define a function $\Omega_{-}^{-1}(x, k)=\Omega_{+}^{+}(x, \tilde{k})$. It is obvious that

$$
\Omega_{-}^{-1}=\left(\begin{array}{c}
\left(H_{-}\right)_{[1]}^{-1} \\
\left(H_{+}\right)_{[2]}^{-1}
\end{array}\right) \text {. }
$$

Then, $\Omega_{-}^{-1}(x, k)$ is a solution of the adjoint spectral problem. On the real axis

$$
\Omega_{-}^{-1}(x, k)=\Omega_{+}^{+}(x, \tilde{k})=F S_{+}^{+} F^{-1} H_{+}^{-1}=F S_{-}^{+} F^{-1} H_{-}^{-1},
$$

and $\operatorname{det} \Omega_{-}^{-1}(x, k)=\bar{a}(\tilde{k}), \Omega_{+}(x, k)$ has an asymptotic expansion as follows:

$$
\Omega_{+}(x, k)=I+\frac{1}{k} \Omega_{+}^{(1)}(x)+O\left(\frac{1}{k^{2}}\right),
$$

and substitute it into the spectral Equation (6). Comparing with powers of $k$, we derive

$$
Q=i\left[\sigma_{3}, \Omega_{+}^{(1)}\right]
$$

In order to solve the coupled KdV Equation (2), we should find the analytic solution $\Omega_{+}$.

\section{Matrix RH Problem}

Through tedious calculation, we obtain RH problem

$$
\Omega_{-}^{-1}(x, k) \Omega_{+}(x, k)=F \Lambda_{0}(k) F^{-1}, \quad \Lambda_{0}=S_{+}^{\dagger} S_{+}=\left(\begin{array}{cc}
1 & \bar{b} \\
a & 1
\end{array}\right),
$$

with $|a|^{2}+|b|^{2}=1, \quad \operatorname{Imk}=0$.

It is easy to know that $\Omega_{-}^{-1}(x, k) \Omega_{+}(x, k)$ only depends on $k$, the $x$-dependence 
being given by the simple exponential function $F$. Moreover, it is obvious that $\Omega_{+}(x, k) \rightarrow F$ for $k \rightarrow \infty$, in view of (12).

In order to obtain the soliton solution of the coupled KdV equation, we suppose that the zeros of $a(k)$ and $\bar{a}(k)$ are simple and finite number. We know that determinants of the matrices $\Omega_{+}$and $\Omega_{-}^{-1}$ are given by $a(k)$ and $\bar{a}(k)$. We assume that

$$
\begin{aligned}
& \operatorname{det} \Omega_{+}\left(k_{j}\right)=0, \quad \operatorname{Im} k_{j}>0, j=1,2, \cdots, N, \\
& \operatorname{det} \Omega_{-}^{-1}\left(\tilde{k}_{j}\right)=0, \quad \operatorname{Im} \tilde{k}_{l}<0, l=1,2, \cdots, N .
\end{aligned}
$$

In this case, the $\mathrm{RH}$ problem (15) with zeros can be solved in view of its regulation.

To obtain the relevant regular problem, let us introduce a rational matrix function

$$
\chi_{j}^{-1}=I+\frac{k_{j}-\tilde{k}_{j}}{k-k_{j}} \Upsilon_{j}, \quad \Upsilon_{j}=\frac{\left|Y_{j}\right\rangle\left\langle Y_{j}\right|}{\left\langle Y_{j} \mid Y_{j}\right\rangle},
$$

where the eigenvector $\left|Y_{j}\right\rangle$ solves $\Omega_{+}\left(k_{j}\right)\left|Y_{j}\right\rangle=0$.

Here $\Upsilon_{j}$ is the rank 1 projector $\Upsilon_{j}^{2}=\Upsilon_{j}$, and $\left\langle Y_{j}|=| Y_{j}\right\rangle^{+}$.

In view of $(11)$, we know that $\operatorname{det} \Omega_{+}(k)\left(k-k_{j}\right)=0$ near the point $k_{j}$. We obtain $\operatorname{det}\left(\Omega_{+} \chi_{j}^{-1}\right) \neq 0$ at the point $k_{j}$. The matrix function $\Omega_{-}^{-1}$ will be regularized by the rational function

$$
\chi_{l}=I-\frac{k_{l}-\tilde{k}_{l}}{k-\tilde{k}_{l}} \Upsilon_{l},
$$

it is easy to know that the matrix $\chi_{l} \Omega_{-}^{-1}$ has no zeros in $\tilde{k}_{l}$.

The regularization of all the other zeros is performed similarly, and eventually we obtain the following representation for the analytic solutions:

$$
\Omega_{ \pm}=\omega_{ \pm} \Gamma, \quad \Gamma=\chi_{N} \chi_{N-1} \cdots \chi_{1},
$$

where the rational matrix function $\Gamma(x, k)$ accumulates all zeros of the $\mathrm{RH}$ problem, while the matrix functions $\omega_{ \pm}$solve the regular RH problem (without zeros)

$$
\omega_{-}^{-1}(x, k) \omega_{+}(x, k)=\Gamma(x, k) F \Lambda_{0}(k) F^{-1} \Gamma^{-1}(x, k),
$$

with $\Lambda_{0}(k)=I$, thus $\Omega_{+}=\Gamma(x, k)$.

The matrix $\Gamma$ will be called the dressing factor. It follows from (16) that the asymptotic expansion for the dressing factor is written as

$$
\Gamma(x, k)=I+\frac{1}{k} \Gamma^{(1)}(x)+O\left(\frac{1}{k^{2}}\right) .
$$

We note that the dress matrix $\Gamma(k)$ can be written as

$$
\begin{aligned}
\Gamma(k) & =\left(I-\frac{k_{N}-\tilde{k}_{N}}{k-\tilde{k}_{N}} \Upsilon_{N}\right) \cdots\left(I-\frac{k_{1}-\tilde{k}_{1}}{k-\tilde{k}_{1}} \Upsilon_{1}\right) \\
& =I-\sum_{l=1}^{N} \frac{k_{l}-\tilde{k}_{1}}{k-\tilde{k}_{1}}\left|x_{l}\right\rangle\left\langle y_{l}\right|, \\
\Gamma^{-1}(k) & =\left(I+\frac{k_{N}-\tilde{k}_{N}}{k-\tilde{k}_{N}} \Upsilon_{N}\right) \cdots\left(I+\frac{k_{1}-\tilde{k}_{1}}{k-\tilde{k}_{1}} \Upsilon_{1}\right)
\end{aligned}
$$




$$
=I+\sum_{l=1}^{N} \frac{k_{l}-\tilde{k}_{l}}{k-\tilde{k}_{l}}\left|y_{l}\right\rangle\left\langle x_{l}\right|
$$

Thus, we derived $2 N$ vectors $\left|x_{j}\right\rangle$ and $\left|y_{j}\right\rangle$ instead of $N$ vectors $\left|Y_{j}\right\rangle$. It is obvious that $\Gamma(k) \Gamma^{-1}(k)=I$ at the point $k=k_{j}$. To avoid divergence at $k \rightarrow k_{j}$, we should pose $\Gamma\left(k_{j}\right)\left|y_{j}\right\rangle\left\langle x_{j}\right|=0$, that is

$$
\left(I-\frac{k_{l}-\tilde{k}_{l}}{k_{j}-\tilde{k}_{l}}\left|y_{l}\right\rangle\left\langle x_{l}\right|\right)\left|y_{j}\right\rangle\left\langle x_{j}\right|=0 .
$$

We note that the matrix $\Gamma(k)$ can be decomposed into the following form:

$$
\Gamma(k)=I-\sum_{j, l=1}^{N} \frac{1}{k-\tilde{k}_{l}}|j\rangle\left(\mathcal{D}^{-1}\right)_{j l}\langle l|, \mathcal{D}_{l j}=\frac{\langle l \mid j\rangle}{k_{j}-\tilde{k}_{l}},
$$

where $\mathcal{D}=\mathcal{D}_{l j}=\left\langle y_{l}\left|\frac{1}{k_{j}-\tilde{k}_{l}}\right| y_{j}\right\rangle$. Similarly,

$$
\Gamma(k)=I-\sum_{j, l=1}^{N} \frac{1}{k-k_{j}}|j\rangle\left(\mathcal{D}^{-1}\right)_{j l}\langle l|,
$$

where $|j\rangle \equiv\left|y_{j}\right\rangle$. In what follows, we rewrite (13) as

$$
Q=i\left[\sigma_{3}, \Gamma^{(1)}(x)\right] \text {. }
$$

Let us differentiate the equation $\Omega_{+}\left(k_{j}\right)|j\rangle=0$ in $x$, and in view of (6), we derive

$$
\partial_{x} \Omega_{+}\left(x, k_{j}\right)|j\rangle+\Omega_{+}\left(x, k_{j}\right)|j\rangle_{x}=i \frac{k_{j}}{2} \Omega_{+}\left(x, k_{j}\right)|j\rangle+\Omega_{+}\left(x, k_{j}\right)|j\rangle_{x}=0,
$$

thus, we have

$$
|j\rangle_{x}=-\frac{1}{2} i k_{j} \sigma_{3}|j\rangle
$$

In the same way, we obtain the evolutionary equation

$$
|j\rangle_{t}=\frac{1}{2} i k_{j}^{2} \sigma_{3}|j\rangle \text {. }
$$

In this end, we establish explicitly the vector $|j\rangle$ as

$$
|j\rangle=\exp \left[\left(-i \frac{1}{2} k_{j} x+i \frac{1}{2} k_{j}^{2}\right) t \sigma_{3}\right]\left|j_{0}\right\rangle,
$$

where $\left|j_{0}\right\rangle$ is a vector integration constant.

Similarly, according to $\langle\tilde{j}| \Omega_{-}^{-1}\left(\tilde{k}_{j}\right)=0$, we obtain the solution

$$
\langle\tilde{j}|=\exp \left[\left(-i \frac{1}{2} \tilde{k}_{j} x+i \frac{1}{2} \tilde{k}_{j}^{2} t\right) \sigma_{3}\right]\left\langle\tilde{j}_{0}\right|,
$$

where $\left\langle\tilde{j}_{0}\right|$ is a vector integration constant.

\section{One Soliton Solution}

We consider the case $N=1$ and pose $k_{1}=\xi+i \eta, \tilde{k}_{1}=\tilde{\xi}+i \tilde{\eta}$. Then, we have 


$$
|1\rangle=\left(\begin{array}{c}
\exp \frac{\eta x}{2} x+\frac{1}{2\left(\xi^{2}-\eta^{2}\right) t}+i\left[-\frac{\xi}{2} x+\xi \eta t\right] p_{1} \\
\exp -\frac{\eta x}{2} x-\frac{1}{2\left(\xi^{2}-\eta^{2}\right) t}-i\left[-\frac{\xi}{2} x+\xi \eta t\right] p_{2}
\end{array}\right),
$$

where, $p_{1}, p_{2}$ are components of the constant vector $\left|1_{0}\right\rangle$.

$$
\langle\tilde{1}|=\left(\begin{array}{c}
\exp \frac{\tilde{\eta} x}{2} x+\frac{1}{2\left(\tilde{\xi}^{2}-\tilde{\eta}^{2}\right) t}+i\left[-\frac{\tilde{\xi}}{2} x+\tilde{\xi} \tilde{\eta} t\right] \tilde{p}_{1} \\
\exp -\frac{\tilde{\eta} x}{2} x-\frac{1}{2\left(\tilde{\xi}^{2}-\tilde{\eta}^{2}\right) t}-i\left[-\frac{\tilde{\xi}}{2} x+\tilde{\xi} \tilde{\eta} t\right] \tilde{p}_{2}
\end{array}\right),
$$

where, $\tilde{p}_{1}, \tilde{p}_{2}$ are components of the constant vector $\left\langle 1_{0}\right|$.

The dress formula (19) reduced to

$$
u=i \Gamma_{12}^{(1)}(x), \quad v=-i \Gamma_{21}^{(1)}(x) .
$$

At the same time, we have $\Gamma=I-\frac{k_{1}-\tilde{k}_{1}}{k-\tilde{k}_{1}} \Upsilon_{1}$, from which, we obtain

$$
\Gamma^{(1)}=-i(\eta+\tilde{\eta}) \frac{|1\rangle\langle\tilde{1}|}{\langle\tilde{1}|| 1\rangle} .
$$

Denoting $z=\eta x+\left(\xi^{2}-\eta^{2}\right) t+\alpha, \quad \kappa=\xi(-x+2 \eta t)+\beta$, thus

$$
|1\rangle=\exp \left[-\frac{\alpha+i \beta}{2}\right]\left(\begin{array}{c}
\exp \frac{z+i \varphi}{2} \\
\exp -\frac{z+i \varphi}{2}
\end{array}\right) \text {. }
$$

In the same way, defining $\tilde{z}=\tilde{\eta} x+\left(\tilde{\xi}^{2}-\tilde{\eta}^{2}\right) t+\tilde{\alpha}, \tilde{\varphi}=\tilde{\xi}(-x+2 \tilde{\eta} t)+\tilde{\beta}$, thus

$$
\langle 1|=\exp \left[-\frac{\tilde{\alpha}+i \tilde{\beta}}{2}\right]\left(\begin{array}{c}
\exp \frac{\tilde{z}+i \tilde{\varphi}}{2} \\
\exp -\frac{\tilde{z}+i \tilde{\varphi}}{2}
\end{array}\right) .
$$

Substituting (31) and (32) into (30), we have

$$
|1\rangle\langle 1|=\exp \left(-\frac{\alpha+\tilde{\alpha}}{2}\right)\left(\begin{array}{cc}
\exp \frac{z+\tilde{z}}{2} & \exp \frac{i \varphi+i \tilde{\varphi}}{2} \\
\exp -\frac{i \varphi+i \tilde{\varphi}}{2} & \exp -\frac{z+\tilde{z}}{2}
\end{array}\right) .
$$

Moreover, $\langle 1 \mid 1\rangle=\exp \left(-\frac{\alpha+\tilde{\alpha}}{2}\right)(\exp z+\exp -\tilde{z})$, hence,

$$
\Gamma^{(1)}=-i(\eta+\tilde{\eta})\left(\begin{array}{cc}
\exp \frac{z+\tilde{z}}{2} & \exp i(\varphi+\tilde{\varphi}) \\
\exp -i(\varphi+\tilde{\varphi}) & \exp -\frac{z+\tilde{z}}{2}
\end{array}\right) \sec (z+\tilde{z}) .
$$

From which, we have the solutions of the coupled KdV Equation (2) 


$$
u=(\tilde{\eta}+\eta) \mathrm{e}^{\mathrm{i} \tilde{\varphi}+\varphi} \sec (z+\tilde{z}), \quad v=-(\tilde{\eta}+\eta) \mathrm{e}^{-i \tilde{\varphi}+\varphi} \sec (z+\tilde{z}) .
$$

Here, $\xi, \tilde{\xi}, \eta$ and $\tilde{\eta}$ determine the soliton velocity and amplitude, respectively, while $\alpha, \beta, \tilde{\alpha}$ and $\tilde{\beta}$ give the initial position and phase of the soliton. In what follows, we plot the graph for $u(x, t)$ in order to analyze the solutions (35). Figure 1 and Figure 2 are the imaginary part and real part of $u(x, t)$, respectively. From the two solution curves, we can see that the difference between the real and imaginary part.

In the same way, we drop the solution curves of $v$ for Figure 3 and Figure 4.

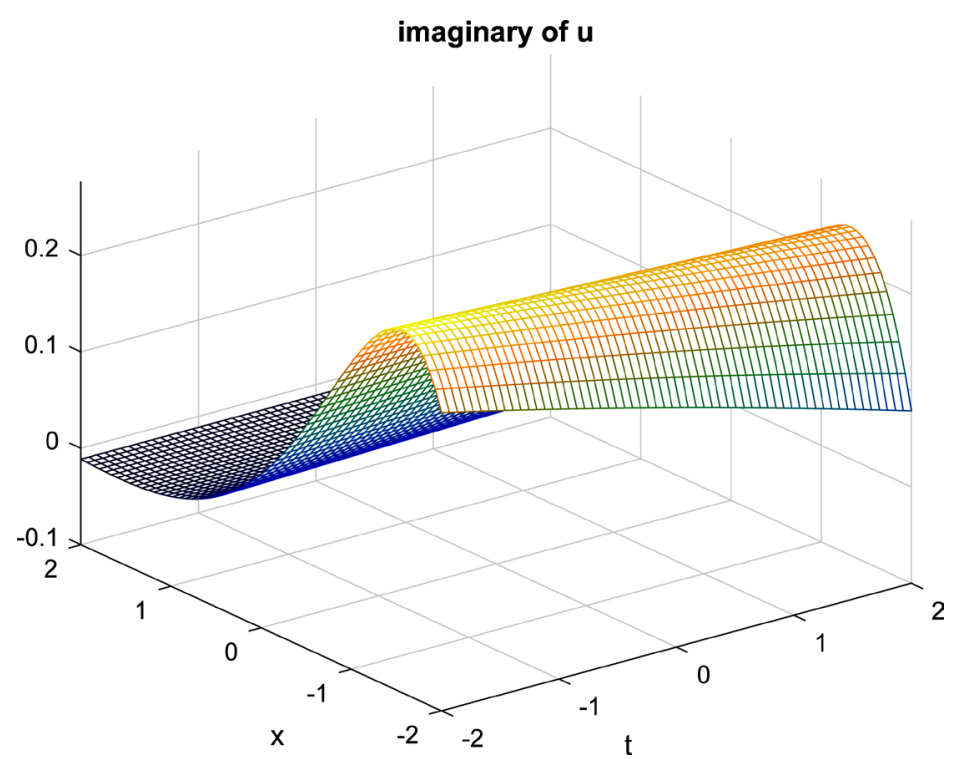

Figure 1. The soliton solution curve of imaginary part of $u(x, t)$ for $\eta=0.01, \tilde{\eta}=0.5$, $\xi=0.05, \tilde{\xi}=0.07, \alpha=0.2, \tilde{\alpha}=0.4, \quad \beta=0.6, \tilde{\beta}=0.9, x \in[-2,2], t \in[-6,6]$.

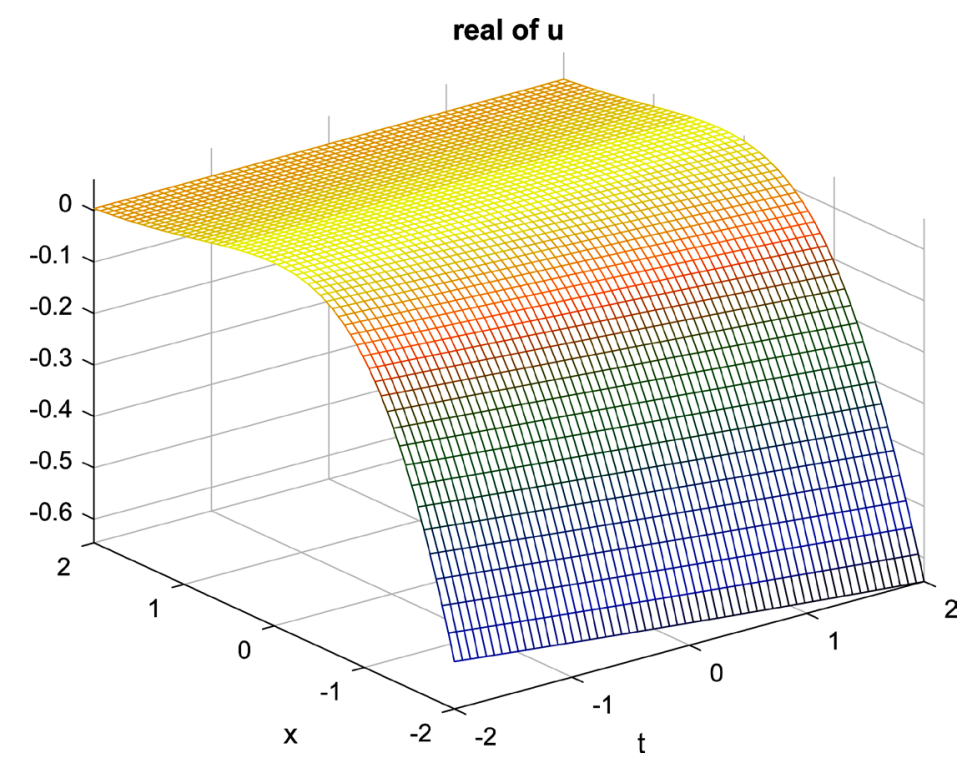

Figure 2. The soliton solution curve of real part of $u(x, t)$ for $\eta=0.01, \tilde{\eta}=0.5, \quad \xi=0.05$, $\tilde{\xi}=0.07, \alpha=0.2, \tilde{\alpha}=0.4, \quad \beta=0.6, \tilde{\beta}=0.9, x \in[-2,2], \quad t \in[-6,6]$. 


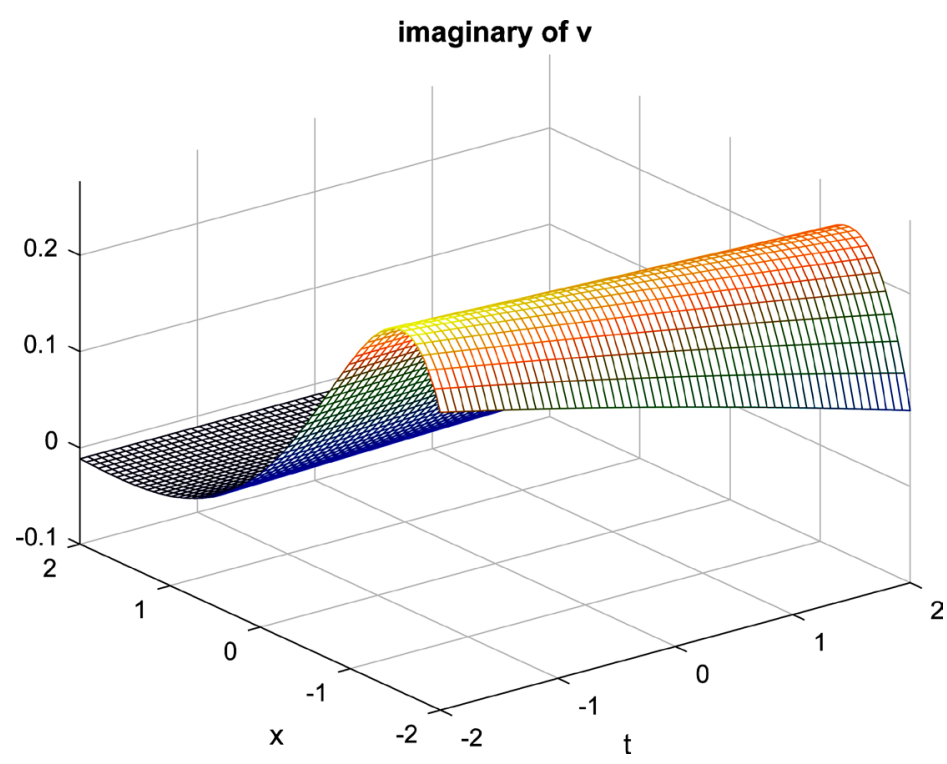

Figure 3. The soliton solution curve of imaginary part of $v(x, t)$ for $\eta=0.01, \tilde{\eta}=0.5$, $\xi=0.05, \tilde{\xi}=0.07, \alpha=0.2, \tilde{\alpha}=0.4, \quad \beta=0.6, \tilde{\beta}=0.9, x \in[-2,2], t \in[-6,6]$.

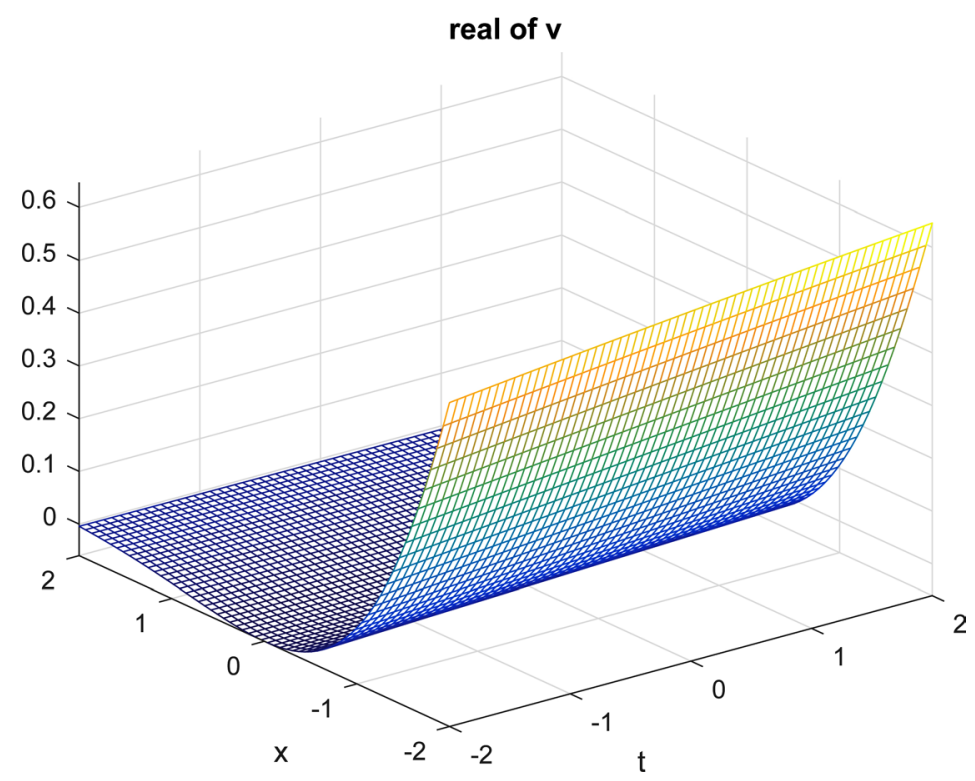

Figure 4. The soliton solution curve of real part of $v(x, t)$ for $\eta=0.01, \tilde{\eta}=0.5, \xi=0.05$, $\tilde{\xi}=0.07, \quad \alpha=0.2, \tilde{\alpha}=0.4, \quad \beta=0.6, \tilde{\beta}=0.9, \quad x \in[-2,2], \quad t \in[-6,6]$.

From the graphs, it is shown that $u$ and $v$ have the similar solution form. The difference exists between the real and imaginary part. In fact, we chose different parameters, and the solution curves between the real part and imaginary part had corresponding changes.

\section{Acknowledgements}

The authors acknowledge the support by National Natural Science Foundation of Chi- 
na (Project No: 11301149), Henan Natural Science Foundation For Basic Research under Grant No: 162300410072, doctor Foundation (D2015001) and Young backbone teachers in Henan province.

\section{References}

[1] Ablowitz, M.J. and Segur, H. (1981) Solitons and the Inverse Scattering Transform. SIMA, Philadelphia.

[2] Geng, X.G., Wu, Y.T. and Cao, C.W. (1999) Quasi-Periodic Solutions of the Modified Kadomtsev-Petviashvili Equation. Journal of Physics A: Mathematical and General, 32, 37333742. http://dx.doi.org/10.1088/0305-4470/32/20/306

[3] Ablowitz, M.J. and Clarkson, P.A. (1991) Solitons, Nonlinear Evolution Equations and the Inverse Scattering. Cambridge University Press, Cambridge.

http://dx.doi.org/10.1017/CBO9780511623998

[4] Gu, C.H., Hu, H.S. and Zhou, H.S. (1999) Darboux Transformation in Soliton Theory and Its Geometric Applications. Shanghai Scientific and Technical Publishers, China.

[5] Geng, H.S. and Tam, H.W. (1999) Darboux Transformation and Soliton Solutions for Generalized Nonlinear Schrodinger Equations. Journal of the Physical Society of Japan, 68, 1508-1542. http://dx.doi.org/10.1143/JPSJ.68.1508

[6] Geng, X.G. (1992) A Hierarchy of Nonlinear Evolution Equations and Corresponding Finite Dimensional Completely Integrable System. Physics Letters A, 180, 375-380. http://dx.doi.org/10.1016/0375-9601(92)90057-S

[7] Deife, P. and Zhou, X. (1993) A Steepest Descent Method for Oscilatory Riemann-Hilbert Problems, Asymptotics for the mKdV Equation. Annals of Mathematics, 137, 295-268.

[8] Claeys, T. and Grava, T. (2009) Universality of the Break-Up Profile for the KdV Equation in the Small Dispersion Limit Using the Riemann-Hilbert Approach. Communications in Mathematical Physics, 286, 979-1009. http://dx.doi.org/10.1007/s00220-008-0680-5

[9] Wang, M.L., Zhou, Y.B. and Li, Z.B. (1996) Application of a Homogeneous Balance Method to Exact Solutions of Nonlinear Equations in Mathematical Physics. Physics Letters A, 216, 67-75. http://dx.doi.org/10.1016/0375-9601(96)00283-6

[10] Tam, H.W., Hu, X.B. and Wang, D.L. (1999) Two Integrable Coupled Nonlinear Systems. Journal of the Physical Society of Japan, 68, 369-379. http://dx.doi.org/10.1143/JPSJ.68.369

[11] Fokas, A.S. and Gelfand, I.M. (1994) Integrability of Linear and Nonlinear Evolution Equations and the Associated Nonlinear Fourier Transforms. Letters in Mathematical Physics, 32, 189-210. http://dx.doi.org/10.1007/BF00750662

[12] Calogero, F. and Degasperis, A. (1982) Solitons and Spectral Transform I. North-Holl, Amsterdam.

[13] Dodd, R.K., Eilbeck, J.C., Gibbon, J.D. and Morris, H.C. (1982) Solitons and Nonlinear Wave Equations. Physics Letters A, 89, 168-170. http://dx.doi.org/10.1016/0375-9601(82)90199-2

[14] Newell, A.C. (1985) Solitons in Mathematics and Physics. SIAM, Philadelphia.

[15] Dotorov, E.V., Matsuka, N.P. and Rothos, V.M. (2003) Perturbation-Induced Radiation by the Ablowitz-Ladik Soliton. Physical Review E, 68, Article ID: 066610. http://dx.doi.org/10.1103/PhysRevE.68.066610

[16] Fokas, A.S. (1997) A Unified Transformation Method for Solving Linear and Certain Nonlinear PDES. Proceedings of the Royal Society of London A, 453, 1441-1443. http://dx.doi.org/10.1098/rspa.1997.0077 
Submit or recommend next manuscript to SCIRP and we will provide best service for you:

Accepting pre-submission inquiries through Email, Facebook, LinkedIn, Twitter, etc. A wide selection of journals (inclusive of 9 subjects, more than 200 journals)

Providing 24-hour high-quality service

User-friendly online submission system

Fair and swift peer-review system

Efficient typesetting and proofreading procedure

Display of the result of downloads and visits, as well as the number of cited articles

Maximum dissemination of your research work

Submit your manuscript at: http://papersubmission.scirp.org/

Or contact am@scirp.org 